\title{
Tunnelling of pulsating strings in deformed Minkowski spacetime
}

\author{
Sergio Giardino ${ }^{1, a}$, Victor Rivelles ${ }^{2, b}$ \\ ${ }^{1}$ Departamento de Física \& Centro de Matemática e Aplicações, Universidade da Beira Interior, Rua Marquês D’Ávila e Bolama, 6200-001 Covilhã, \\ Portugal \\ ${ }^{2}$ Instituto de Física, Universidade de São Paulo, Caixa Postal 66318, 05315-970 São Paulo, SP, Brazil
}

Received: 18 December 2015 / Accepted: 10 April 2016 / Published online: 27 April 2016

(c) The Author(s) 2016. This article is published with open access at Springerlink.com

\begin{abstract}
Using the WKB approximation we analyse the tunnelling of a pulsating string in deformed Minkowski spacetime.
\end{abstract}

\section{Introduction}

There is a huge body of evidence that the AdS/CFT correspondence holds true for strings in $A d S_{5} \times S^{5}$ and $\mathcal{N}=4$ super Yang-Mills theory in four dimensions. For instance, the energy of spinning and rotating strings matches the anomalous dimension of operators in the gauge theory in the range where they can be compared. Integrability on both sides of the correspondence also provides further support for the correspondence [1]. It is also important to test the correspondence in situations with less supersymmetry where the gauge theories have deformed potentials leading to marginally deformed $\mathcal{N}=2$ or $\mathcal{N}=1$ supersymmetric gauge theories [2,3]. The gravitational dual of such theories have a deformed fivesphere characterised by a real parameter $\gamma$ and the dilaton and some RR and NS-NS fields are also present [4]. In this situation both sides of the correspondence also have integrable structures [5]. Spinning and rotating strings have also been considered in such a deformed context and they confirm the correspondence whenever they can be compared [5-16].

There is a class of string configurations, pulsating strings, which has not received much attention since its dual operator is not completely understood. They have been analysed in $A d S_{5} \times S^{5}$ [17-27], $A d S_{4} \times C P^{3}$ [28,29] and other backgrounds [30-37], and more recently they have been studied in the deformed case as well [38]. Since the string presents a periodic motion its dynamics can be characterised by its oscillation number. It is not one of the string charges but it is quite useful to parametrise its behaviour [23,38,39]. At the quantum level it is an adiabatic invariant so it provides infor-

\footnotetext{
a e-mail: p12@ubi.pt

b e-mail: rivelles@fma.if.usp.br
}

mation as regards the semi-classical regime for higher values of the oscillation number. In [38] we analysed pulsating strings in deformed Minkowski spacetime and in deformed $A d S_{5} \times S^{5}$ for a small deformation. We have found the classical energy in terms of the oscillation number in the high and low energy limits. For high energy we performed the quantisation of the highly excited string states to second order in perturbation theory and found that the oscillation number has to be even. In the low energy case we found a new term, proportional to $\gamma$, which is not present in the classical case.

In order to analyse the classical dynamics of the pulsating string we introduced an effective potential which captures all relevant information as regards the deformed background. When the string pulsates on the deformed five-sphere its effective potential grows smoothly as one of the angles increase from zero to $\pi / 2$ and the oscillation number can be expressed in terms of complete elliptic integrals [38]. In the case of deformed Minkowski spacetime the string pulsates along the radial direction and the effective potential starts growing from the origin until it reaches a maximum value of $m^{2} /(2 \gamma)$ at $r^{2}=1 / \gamma$ and then goes back to zero far away from the origin (see Fig. 1). It is clear that at low energies or small deformation the string has a periodic motion that can be quantised perturbatively as done in [38]. However, since the potential has a maximum, it is possible for the string to tunnel through the potential barrier and the computation of the transition rate for such a process is the main goal of this paper.

A non-perturbative phenomenon like tunnelling may be studied semi-classically using the WKB approximation whenever the amplitude or the phase of the wave function is taken to be slowly changing. The WKB method has been applied in several situation involving strings [40-44] and here it will be used to analyse the behaviour of a pulsating string in deformed Minkowski spacetime.

This paper is organised as follows. In Sect. 2 the pulsating string in deformed ten-dimensional Minkowski spacetime will be briefly described. In Sect. 3 we will use the WKB 
technique to calculate the transition rate for the pulsating string to tunnel through the potential. In Sect. 4 we will analyse the classical stability of the pulsating string and show that for small deformation it is stable. We then present some conclusions in the last section.

\section{Pulsating strings in deformed Minkowski spacetime}

The energy of a semi-classical pulsating string in tendimensional Minkowski spacetime and in $\operatorname{Ad}_{5} \times S^{5}$ was computed in terms of its oscillation number in [19] while for the case of a deformed Minkowski spacetime and deformed $A d S_{5} \times S^{5}$ the energy was found in [38]. We will briefly review the case of deformed Minkowski spacetime. Lunin and Maldacena [4] found a technique to build deformed supergravity backgrounds which have a $U(1) \times U(1)$ global symmetry required by the deformed gauge theory. When applied to the ten-dimensional Minkowski spacetime it gives the deformed background

$$
\begin{aligned}
\mathrm{d} s^{2}= & \eta_{\mu \nu} \mathrm{d} x^{\mu} \mathrm{d} x^{\nu}+\sum_{i=1}^{3}\left(\mathrm{~d} r_{i}^{2}+\mathcal{G} r_{i}^{2} \mathrm{~d} \phi_{i}^{2}\right) \\
& +\gamma^{2} r_{1}^{2} r_{2}^{2} r_{3}^{2} \mathcal{G}\left(\sum_{i=1}^{3} \mathrm{~d} \phi_{i}\right)^{2}, \\
\mathcal{G}^{-1}= & 1+\gamma^{2}\left(r_{1}^{2} r_{2}^{2}+r_{1}^{2} r_{3}^{2}+r_{2}^{2} r_{3}^{2}\right),
\end{aligned}
$$

where a four-dimensional Minkowski spacetime is left undeformed and the remaining six-dimensional space with coordinates $\left(r_{i}, \phi_{i}\right), i=1,2,3$, has a deformation parameter $\gamma$. When $\gamma$ vanishes we recover the ten-dimensional Minkowski spacetime. The dilaton and the $B_{2}$ field have non-trivial configurations given by

$$
\begin{aligned}
B_{2}= & \gamma \mathcal{G}\left(r_{1}^{2} r_{2}^{2} \mathrm{~d} \phi_{1} \wedge \mathrm{d} \phi_{2}+r_{2}^{2} r_{3}^{2} \mathrm{~d} \phi_{2} \wedge \mathrm{d} \phi_{3}\right. \\
& \left.+r_{1}^{2} r_{3}^{2} \mathrm{~d} \phi_{1} \wedge \mathrm{d} \phi_{3}\right), \\
e^{2 \Phi}= & \mathcal{G} .
\end{aligned}
$$

The deformed Minkowski background is a supergravity solution [4], but only its bosonic part is known. The fermionic part is not relevant for our purposes. The parametrisation for a pulsating string used in [38] is not convenient in analysing stability issues. Instead we will take a string at the origin of Minkowski spacetime with

$t=\sqrt{2} \kappa \tau, \quad r_{1}=r_{2}=r(\tau), \quad r_{3}=0$,

$\phi_{1}=\phi_{2}=m \sigma, \quad \phi_{3}=0$,

where $m$ is the string winding number. This ansatz is compatible with the classical equations of motion for the pulsating string and is suitable for the classical stability analysis of Sect. 4. It corresponds to a string dynamically equivalent to the simplest and well-known pulsating strings studied in [19,38]. Using (2) in (1) we get

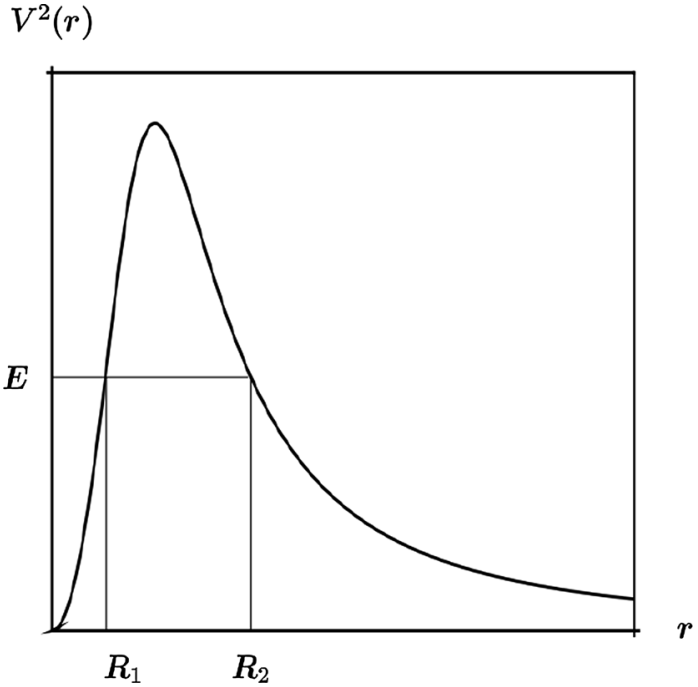

Fig. 1 The effective potential in deformed Minkowski spacetime for $m^{2}=20$ and $\gamma=1$

$\mathrm{d} s^{2}=-\mathrm{d} t^{2}+2\left(\mathrm{~d} r^{2}+\mathcal{G} r^{2} \mathrm{~d} \phi^{2}\right)$,

$\mathcal{G}^{-1}=1+\gamma^{2} r^{4}$.

For this choice there is no coupling of the string to the $B_{2}$ field. Then the Nambu-Goto action becomes

$S=-m \int \mathrm{d} \tau r \sqrt{\mathcal{G}\left(1-\dot{r}^{2}\right)}$,

where we set the string tension equal to one.

We can then find that the radial canonical momentum $\Pi$ and the squared canonical Hamiltonian is given by

$H^{2}=\Pi^{2}+\frac{m^{2} r^{2}}{1+\gamma^{2} r^{4}}$.

We can identify an effective potential

$V^{2}(r)=\frac{m^{2} r^{2}}{1+\gamma^{2} r^{4}}$

which governs the string dynamics. The potential has a maximum at $r=1 / \sqrt{\gamma}$ where its value is $m^{2} /(2 \gamma)$ (see Fig. 1) providing a barrier for a pulsating string trapped in the region $r<1 / \sqrt{\gamma}$. For a particle of energy $E^{2}<m^{2} /(2 \gamma)$ there are two points where its radial velocity vanishes

$R_{1,2}^{2}=\frac{m^{2}}{2 \gamma^{2} E^{2}}\left(1 \mp \sqrt{1-\frac{4 \gamma^{2} E^{4}}{m^{4}}}\right)$.

This means that the pulsating string can, in principle, tunnel from $r<R_{1}$, through the classically forbidden region of the potential, and escape to the classically allowed region $r>R_{2}$. 
The equation of motion can be integrated in terms of elliptic functions and the energy can be found in terms of the oscillation number $\mathcal{N}=\oint \Pi \mathrm{d} r / 2 \pi$. For more details see [38].

\section{String tunnelling}

To apply the WKB method we assume that the wave function depends only on $r$ so that we can take for $\Pi^{2}$ the radial part of the Laplacian

$\Pi^{2}=-\frac{\hbar^{2}}{\sqrt{-g}} \frac{\mathrm{d}}{\mathrm{d} r}\left(\sqrt{-g} \frac{\mathrm{d}}{\mathrm{d} r}\right)$

where $\sqrt{-g}=r^{(d-1)} \mathcal{G}$ is the determinant of the metric. Here we left the number of dimensions of the deformed part of the space $d$ arbitrary since we want to consider the general situation. For the full ten-dimensional case $d=6$. Then the Schrodinger equation reads

$-\frac{\hbar^{2}}{r^{(d-1)}}\left(r^{(d-1)} \Psi^{\prime}\right)^{\prime}+\frac{4 \hbar^{2} \gamma^{2} r^{3}}{1+\gamma^{2} r^{4}} \Psi^{\prime}+\frac{m^{2} r^{2}}{1+\gamma^{2} r^{4}} \Psi=E^{2} \Psi$,

and the WKB ansatz is
If we choose

$c_{i}=\sqrt[3]{\frac{2 E^{2}}{\hbar^{2} R_{i}} \frac{1-\gamma^{2} R_{i}^{4}}{1+\gamma^{2} R_{i}^{4}}}$,

we can reduce (12) to the Airy equation by the change of variable $\Psi_{R_{i}}(x)=\exp \left(-a_{i} x / 2\right) \chi_{i}(x)$, which yields

$\chi_{i}^{\prime \prime}-\left(x+\frac{a_{i}^{2}}{4}\right) \chi_{i}=0$

Then, near $R_{1}$ and $R_{2}$, we have

$\Psi_{R_{i}}(x)=e^{-\frac{a_{i} x}{2}}\left(a_{i} A i\left(x+\frac{a_{i}^{2}}{4}\right)+b_{i} B i\left(x+\frac{a_{i}^{2}}{4}\right)\right)$,

where $a_{i}$ and $b_{i}$ are integration constants and $A i(x)$ and $B i(x)$ are the two linearly independent Airy functions.

To match the WKB and the Airy solutions around $R_{i}$ we must make sure that they have the same functional form for large $|x|$. Around $R_{1}$ we find that in the WKB solution $\int_{r}^{R_{1}} p(r) \mathrm{d} r=2 / 3 \hbar(-x)^{3 / 2}$ for $x<0$, while for

$\Psi^{W K B}(r)= \begin{cases}\frac{1}{\sqrt{r^{(d-1)} \mathcal{G}(r) p(r)}}\left(A e^{\frac{i}{\hbar} \int_{r}^{R_{1}} \mathrm{~d} r p(r)}+B e^{-\frac{i}{\hbar} \int_{r}^{R_{1}} \mathrm{~d} r p(r)}\right), & r<R_{1}, \\ \frac{1}{\sqrt{r^{(d-1)} \mathcal{G}(r)|p(r)|}}\left(C e^{\frac{1}{\hbar} \int_{R_{1}}^{r} \mathrm{~d} r|p(r)|}+D e^{-\frac{1}{\hbar} \int_{R_{1}}^{r} \mathrm{~d} r|p(r)|}\right), & R_{1}<r<R_{2}, \\ \frac{1}{\sqrt{r^{(d-1)} \mathcal{G}(r) p(r)}} F e^{\frac{i}{\hbar} \int_{R_{2}}^{r} \mathrm{~d} r p(r)}, & r>R_{2},\end{cases}$

where $A, B, C, D$ and $F$ are constants and $p(r)=$ $\sqrt{E^{2}-V^{2}(r)}$.

The WKB approximation does not hold in the neighbourhood of $r=R_{1}$ and $r=R_{2}$ because $p(r)$ vanishes at these points. To avoid this problem we will consider solutions of (10) around these two points. To this end we introduce coordinates $x=c_{i}\left(r-R_{i}\right), i=1,2$, where $R_{i}$ stands for $R_{1}$ or $R_{2}$, and we find that (10) reduces to

$\Psi_{R_{i}}^{\prime \prime}+a_{i} \Psi_{R_{i}}^{\prime}-x \Psi_{R_{i}}=0$

where $\Psi_{R_{i}}$ is the wave function around $R_{i}$ and

$a_{i}=\frac{d-5}{c_{i} R_{i}}\left(1+\frac{4}{d-5} \frac{E^{2}}{m^{2} R_{i}^{2}}\right)$. $x>0, \int_{r}^{R_{1}} p(r) \mathrm{d} r=-2 / 3 \hbar x^{3 / 2}$. The Airy functions go like $e^{ \pm 2 / 3 x^{3 / 2}}$ for $x<0$ and $\cos \left(2 / 3(-x)^{3 / 2}-\pi / 4\right)$ and $\sin \left(2 / 3(-x)^{3 / 2}-\pi / 4\right)$ for $x>0$. Similar expressions hold for the solutions around $R_{2}$. Matching the solutions we find that around $R_{1}$ we have

$a_{1}=2\left(\frac{1+\gamma^{2} R_{1}^{4}}{\hbar c_{1} R_{1}^{d-1}}\right)^{1 / 2} D$,
$b_{1}=\left(\frac{1+\gamma^{2} R_{1}^{4}}{\hbar c_{1} R_{1}^{d-1}}\right)^{1 / 2} C$,

$A=\left(-i D+\frac{C}{2}\right) e^{i \pi / 4}$,

$B=\left(i D+\frac{C}{2}\right) e^{-i \pi / 4}$, 
while around $R_{2}$ we find

$a_{2}=2\left(\frac{1+\gamma^{2} R_{1}^{4}}{\hbar c_{2} R_{2}^{d-1}}\right)^{1 / 2} e^{\mathcal{P} / \hbar} C$,

$b_{2}=\left(\frac{1+\gamma^{2} R_{1}^{4}}{\hbar c_{2} R_{2}^{d-1}}\right)^{1 / 2} e^{-\mathcal{P} / \hbar} D$

$a_{2}=e^{i \pi / 4}\left(\frac{1+\gamma^{2} R_{1}^{4}}{\hbar c_{2} R_{2}^{d-1}}\right)^{1 / 2} F$,

$b_{2}=-i a_{2}$,

where $\mathcal{P}=\int_{R_{1}}^{R_{2}}|p(r)| \mathrm{d} r$. We can now compute $|F / A|^{2}$ to find

$\left|\frac{F}{A}\right|^{2}=\left(\frac{1}{4} e^{-\mathcal{P} / \hbar}-e^{\mathcal{P} / \hbar}\right)^{-2}=\frac{e^{-2 \mathcal{P} / \hbar}}{\left(1-\frac{1}{4} e^{-2 \mathcal{P} / \hbar}\right)^{2}}$.

Notice that all explicit dependence on $d$ has gone away and only $\mathcal{P}$ depends on the dimension through the potential $V^{2}(r)$.

To find the tunnelling probability we have to consider the probability current $i\left(\Psi^{*} \nabla \Psi-\Psi \nabla \Psi^{*}\right)$ in the deformed Minkowski spacetime (1). Taking only the radial component and integrating it with the proper measure we find that the square root factors in (11) precisely cancel the measure factors so that in the region $r<R_{1}$ it gives $|B|^{2}-|A|^{2}$. Unitarity is then respected since (17)-(24) imply that $|B|^{2}-|A|^{2}=$ $|F|^{2}$. This means that the tunnelling amplitude $T=|F / A|^{2}$ is given by (25).

The tunnelling amplitude (25) depends only on $\mathcal{P}=$ $\int_{R_{1}}^{R_{2}}\left|\sqrt{E^{2}-V^{2}(r)}\right| \mathrm{d} r$, with $V^{2}(r)$ given by (7). This integral is quite complicated but can be performed when the deformation is small. To that end we redefine $r$ as $\tilde{r}=\sqrt{\gamma} r$ so that for $\gamma<<1$ we have

$$
\begin{aligned}
& \tilde{R}_{1}=\sqrt{\gamma} R_{1}=\sqrt{\gamma} \frac{E}{m}\left(1+\frac{1}{2} \gamma^{2} \frac{E^{4}}{m^{4}}\right), \\
& \tilde{R}_{2}=\sqrt{\gamma} R_{2}=\frac{m}{\sqrt{\gamma} E}\left(1-\frac{1}{2} \gamma^{2} \frac{E^{4}}{m^{4}}\right) .
\end{aligned}
$$

Notice that $\tilde{R}_{1} \tilde{R}_{2}=1$; from the condition $E^{2}<m^{2} /(2 \gamma)$ we find that $\tilde{R}_{1}<1$. Calling $\tilde{R}=\tilde{R}_{1}$, we find that

$$
\mathcal{P}=\frac{E}{\sqrt{\gamma} \tilde{R}} \int_{\tilde{R}}^{1 / \tilde{R}} \sqrt{\frac{\left(1-\tilde{R}^{2} \tilde{r}^{2}\right)\left(\tilde{r}^{2}-\tilde{R}^{2}\right)}{1+\tilde{r}^{4}}} \mathrm{~d} \tilde{r} .
$$

We can now split the integral from $\tilde{R}$ to $1 / \tilde{R}$ into two integrals, one from $\tilde{R}$ to 1 and the other from 1 to $1 / \tilde{R}$. For the second integral we can again change the integration variable $\tilde{r}$ to $1 / \tilde{r}$ so that

$$
\mathcal{P}=\frac{E}{\sqrt{\gamma} \tilde{R}} \int_{\tilde{R}}^{1}\left(1+\frac{1}{\tilde{r}^{2}}\right) \sqrt{\frac{\left(1-\tilde{R}^{2} \tilde{r}^{2}\right)\left(\tilde{r}^{2}-\tilde{R}^{2}\right)}{1+\tilde{r}^{4}}} \tilde{r} \mathrm{~d} \tilde{r} .
$$

We can then expand the two factors in the numerator inside the square root and perform the integrals. Keeping only the leading terms in $\gamma$ we find that

$\mathcal{P}=\frac{m}{2 \gamma}\left|\ln \left(\frac{\sqrt{\gamma} E}{m}\right)\right|$,

so that when the deformation vanishes the transition amplitude also vanishes as expected.

\section{Classical stability}

As show in the previous section a pulsating string can tunnel through the potential barrier and this naturally raises questions about its classical stability. It is well known that spinning strings in anti-de Sitter spaces are classically unstable for large spin [45]. Pulsating strings, on the other side, have better stability properties than spinning strings, as shown in [46]. In the following we will analyse the stability properties of pulsating strings in deformed Minkowski spacetime. We will apply the technique developed by Larsen and Frolov [47] and we will show that when the deformation is small the classical pulsating string is stable.

We start with the Polyakov action in curved spacetime regarding the string coordinates and the worldsheet metric as independent variables. Following [47] the first variation of the Polyakov action gives

$$
\begin{aligned}
\delta S_{P}= & -\frac{\sqrt{\lambda}}{2} \int \mathrm{d} \xi^{2}\left[\left(\frac{1}{2} h^{a b} G-G^{a b}\right) \delta h_{a b}\right. \\
& \left.-2 g_{\mu \nu}\left(\square X^{\nu}+h^{a b} \Gamma_{\lambda \kappa}^{\nu} X_{, a}^{\lambda} X_{, b}^{\kappa}\right) \delta X^{\mu}\right],
\end{aligned}
$$

where $G_{a b}=g_{\mu \nu} X_{, a}^{\mu} X_{, b}^{\nu}$ is the induced metric, $G=h^{a b} G_{a b}$ and $\xi^{a}$ are the worldsheet coordinates. In order to get the second variation of the action, a general perturbation $\delta X^{\mu}$ is decomposed into normal and tangential components on the worldsheet as

$\delta X^{\mu}=X_{, a}^{\mu} \delta X^{a}+n_{r}^{\mu} \delta X^{r}, \quad r=2, \ldots 9$,

where $\delta X^{a}$ is the tangential perturbation and $\delta X^{r}$ is the normal variation. The normal vectors $n_{a}^{\mu}$ are orthonormal to each other and obey

$g_{\mu \nu} n_{r}^{\mu} n_{s}^{\nu}=\delta_{r s}$,

$g_{\mu \nu} X_{, a}^{\mu} n_{r}^{\nu}=0$.

The non-physical perturbations are then excluded by the choice $\delta X^{a}=0$. We now introduce the second fundamental 
form $\Omega_{r a b}$ and the normal fundamental form $\mu_{r s a}$ defined, respectively, as

$$
\begin{aligned}
& \Omega_{r a b}=g_{\mu \nu} n_{r}^{\mu} \nabla_{a} X_{, b}^{v}, \\
& \mu_{r s a}=g_{\mu \nu} n_{r}^{\mu} \nabla_{a} n_{s}^{v},
\end{aligned}
$$

where $\nabla_{a}=x_{, a}^{\rho} \nabla_{\rho}$, with $\nabla_{\rho}$ being the spacetime covariant derivative. After these definitions the second variation of the action is found to be

$$
\begin{aligned}
\delta^{2} S_{P}= & -\frac{\sqrt{\lambda}}{2} \int \mathrm{d} \xi^{2} \sqrt{-h} \\
& \times\left[\delta h_{a b}\left(2 G^{b c} h^{a d}-\frac{1}{2} h^{a d} h^{b c} G-\frac{1}{2} h^{a b} G^{c d}\right) \delta h_{c d}\right. \\
& +2 \delta h_{a b} h^{a c} h^{b d} \Omega_{r c d} \delta x^{r} \\
& -2 \delta X^{r}\left(\delta_{r s} \square-h^{a b} g_{\mu \nu} \nabla_{a} n_{r}^{\mu} \nabla_{b} n_{s}^{v}-2 h^{a b} \mu_{r s a} \partial_{b}\right. \\
& \left.\left.-h^{a b} X_{, a}^{\mu} X_{, b}^{v} R_{\mu \kappa \lambda \nu} n_{r}^{\kappa} n_{s}^{\lambda}\right) \delta X^{s}\right],
\end{aligned}
$$

where $R_{\mu \kappa \lambda v}$ is the Riemann tensor. Taking into account that the variation of the internal metric is related to the variation of the spacetime coordinates by

$$
\Omega_{a b r} \delta X^{r}=-\frac{1}{4}\left(G \delta h_{a b}-h_{a b} G_{c d} \delta h_{c d}\right),
$$

it can be shown that the second variation of the action is

$$
\begin{aligned}
\delta^{2} S_{P}= & -\frac{\lambda}{2} \int \mathrm{d} \xi^{2} \sqrt{-h} \delta X^{r}\left(\delta_{r s} \square-2 h^{a b} \mu_{r s b} \partial_{a}\right. \\
& -\delta^{t u} h^{a b} \mu_{r t a} \mu_{s u b}-\frac{2}{G} h^{a c} h^{b d} \Omega_{a b r} \Omega_{c d s} \\
& \left.-h^{a b} X_{, a}^{\mu} X_{, b}^{v} R_{\mu \kappa \lambda \nu} n_{r}^{\kappa} n_{s}^{\lambda}\right) \delta X^{s} .
\end{aligned}
$$

The equation of motion for the perturbation is then given by

$$
\begin{gathered}
\square \delta X_{r}-2 h^{a b} \mu_{r s a} \partial_{b} \delta X^{s}-h^{a b} \nabla_{a} \mu_{r s b} \delta X^{s} \\
-\left(\delta^{t u} h^{a b} \mu_{r t a} \mu_{s u b}+\frac{2}{G} h^{a c} h^{b d} \Omega_{a b r} \Omega_{c d s}\right. \\
\left.+h^{a b} X_{, a}^{\mu} X_{, b}^{v} R_{\mu \kappa \lambda v} n_{r}^{\kappa} n_{s}^{\lambda}\right) \delta X^{s}=0 .
\end{gathered}
$$

Now we will particularise the stability analysis to the deformed Minkowski space (1) using (2). The equation of motion for $r(\tau)$ is

$$
\dot{r}^{2}=\kappa^{2}-m^{2} r^{2} \mathcal{G} .
$$

For a small deformation, $\gamma^{2} \ll 1$, it reduces to $\dot{r}^{2}=\kappa^{2}-$ $m^{2} r^{2}+\gamma^{2} m^{2} r^{6}$ and it oscillates between $r=0$ and $r=r_{-}$, where $r_{-}^{2}=\kappa^{2} / m^{2}+\gamma^{2} \kappa^{6} / m^{6}$. Then the motion is periodic with amplitude $\frac{\kappa}{m}\left(1+\frac{1}{2} \gamma^{2} \frac{\kappa^{4}}{m^{4}}\right)$. We can find explicit solutions like $r(\tau)=\frac{\kappa}{m}\left(1+\frac{1}{2} \gamma^{2} \frac{\kappa^{4}}{m^{4}}\right) \sin \left(m \tau+\gamma^{2} \frac{\kappa^{4}}{m^{4}} g(m \tau)\right)$,

$g(m \tau)=-\frac{15}{16} m \tau+\frac{1}{4} \sin (2 m \tau)-\frac{1}{64} \sin (4 m \tau)$,

but they will not be needed for the stability analysis.

The induced metric is given by $G_{00}=-G_{11}=-2 m^{2} r^{2} \mathcal{G}$ and the orthogonality of the normal basis (33) requires

$\sqrt{2} \kappa n_{r}^{t}-\left(n_{r}^{r_{1}}+n_{r}^{r_{2}}\right) \dot{r}=0$,

$n_{r}^{\phi_{1}}+n_{r}^{\phi_{2}}=0$.

The choice of the normal vectors satisfying the first constraint in (41) requires some work. So let us denote our basis vectors

$n_{r}^{\mu}=\left(n_{r}^{t}, n_{r}^{x_{i}}, n_{r}^{r_{i}}, n_{r}^{\phi_{i}}\right), \quad i=1,2,3$.

Consider the first constraint for the normal vectors of the form

$n_{r}^{\mu}=\left(n_{r}^{t}, 0,0,0, n_{r}^{r_{1}}, n_{r}^{r_{2}}, 0,0,0,0\right)$,

which are chosen to be non-trivial for $r=2,3$. Using the constraint and the orthogonality condition, we obtain

$\left[\left(1-\alpha^{2}\right) n_{3}^{r_{1}}-\alpha^{2} n_{3}^{r_{2}}\right] n_{2}^{r_{1}}+\left[\left(1-\alpha^{2}\right) n_{3}^{r_{2}}-\alpha^{2} n_{3}^{r_{1}}\right] n_{2}^{r_{2}}=0$,

where $\alpha=\frac{\dot{r}}{\sqrt{2} \kappa} \leq 1$. The constraint also allows us to rewrite the normalisation as

$$
\left(1-\alpha^{2}\right)\left(n_{r}^{r_{1}}+n_{r}^{r_{2}}\right)^{2}-2 n_{r}^{r_{1}} n_{r}^{r_{2}}=1 .
$$

Matching the norm of the vectors and using (44), we obtain

$n_{2}^{r_{1}}= \pm \frac{\left(1-\alpha^{2}\right) n_{3}^{r_{2}}-\alpha^{2} n_{3}^{r_{1}}}{\sqrt{1-2 \alpha^{2}}}$.

By setting $n_{3}^{r_{1}}=-n_{3}^{r_{2}}=\frac{1}{\sqrt{2}}$ we finally find

$$
\begin{aligned}
n_{2}= & \frac{\kappa}{m r \mathcal{G}}\left(\frac{\dot{r}}{\kappa}, 0,0,0, \frac{1}{\sqrt{2}}, \frac{1}{\sqrt{2}}, 0,0,0,0\right), \\
n_{3}= & \left(0,0,0,0, \frac{1}{\sqrt{2}},-\frac{1}{\sqrt{2}}, 0,0,0,0\right), \\
n_{4}= & (0,1,0,0,0,0,0,0,0,0), \\
n_{5}= & (0,0,1,0,0,0,0,0,0,0), \\
n_{6}= & (0,0,0,1,0,0,0,0,0,0), \\
n_{7}= & (0,0,0,0,0,0,1,0,0,0), \\
n_{8}= & \left(0,0,0,0,0,0,0, \frac{1}{\sqrt{\left(r_{1}^{2}+r_{2}^{2}\right) \mathcal{G}}},\right. \\
& \left.-\frac{1}{\sqrt{\left(r_{1}^{2}+r_{2}^{2}\right) \mathcal{G}}}, 0\right),
\end{aligned}
$$


$n_{9}=\left(0,0,0,0,0,0,0,0,0, \frac{1}{r_{3}}\right)$.

We notice that there are only seven basis vectors since we gauge fixed three coordinates, $t, \phi_{1}$ and $\phi_{2}$.

The fundamental forms can now be found. Using the ansatz (2) we get

$\mu_{r s 0}=0$,

$\mu_{r s 1}=m r \mathcal{G}\left[n_{r}^{\phi_{1}}\left(n_{s}^{r_{1}}-n_{s}^{r_{2}}\right)-n_{s}^{\phi_{1}}\left(n_{r}^{r_{1}}-n_{r}^{r_{2}}\right)\right]$,

$\Omega_{r 01}=0$,

$\Omega_{r 11}=-m^{2}\left(n_{r}^{r_{1}}+n_{r}^{r_{2}}\right) r \mathcal{G}^{2}\left(1-\gamma^{2} r^{4}\right)$,

$\Omega_{r 00}=3\left(n_{r}^{r_{1}}+n_{r}^{r_{2}}\right) \ddot{r}$.

Using the expressions for the basis vectors (47) and $\ddot{r}=$ $m^{2} r \mathcal{G}(1-2 \mathcal{G})$ we obtain the non-vanishing components,

$\mu_{381}=-m \sqrt{\mathcal{G}}, \quad \Omega_{200}=3 \Omega_{211}$,

$\Omega_{211}=\kappa m \sqrt{2 \mathcal{G}}(1-2 \mathcal{G})$.

In order to calculate the curvature term in the equations of motion for the perturbations (38) we use the ansatz (2) to find the non-vanishing components of the curvature tensor,

$R_{r_{1} \phi_{1} r_{1} \phi_{1}}=R_{r_{1} \phi_{1} r_{2} \phi_{1}}=R_{r_{1} \phi_{2} r_{2} \phi_{2}}=R_{r_{2} \phi_{2} r_{2} \phi_{2}}=3 \gamma^{2} r^{4} \mathcal{G}^{3}$,

$R_{r_{1} \phi_{2} r_{1} \phi_{2}}=R_{r_{2} \phi_{1} r_{2} \phi_{1}}=\gamma^{2} r^{4}\left(1-2 r^{4} \gamma^{2}\right) \mathcal{G}^{3}$,

$R_{r_{3} \phi_{1} r_{3} \phi_{1}}=R_{r_{3} \phi_{2} r_{3} \phi_{2}}=-R_{r_{3} \phi_{1} r_{3} \phi_{2}}=\gamma^{2} r^{4} \mathcal{G}^{2}$,

$R_{\phi_{1} \phi_{2} \phi_{1} \phi_{2}}=2 \gamma^{2} r^{6} \mathcal{G}^{4}$.

The curvature dependent terms then become

$$
\begin{aligned}
G^{a b} X_{, a}^{\mu} X_{, b}^{\nu} R_{\mu \rho \sigma \nu} n_{r}^{\rho} n_{s}^{\sigma} \delta X^{s} & \begin{array}{ll}
\frac{\gamma^{2} \kappa^{2}}{m^{2}}(1-6 \mathcal{G}) \delta X_{2}, & r=2, \\
-\gamma^{2} r^{2} \mathcal{G}(1-6 \mathcal{G}) \delta X_{3}, & r=3, \\
\frac{\gamma^{2}}{m^{2}}\left[\dot{r}^{2}(8-\mathcal{G})-2 \kappa^{2} \mathcal{G}\right] \delta X_{8}, & r=8, \\
0, & r=4,5,6,7,9 .
\end{array}
\end{aligned}
$$

Finally, we have to take into account the Kalb-Ramond term,

$S_{B}=-\sqrt{\lambda} \int \mathrm{d} \xi^{2} B_{\mu \nu} \epsilon^{a b} X_{, a}^{\mu} X_{, b}^{\nu}$.

Its second variation gives

$$
\begin{aligned}
\delta^{2}( & \left.B_{\mu \nu} \epsilon^{a b} X_{, a}^{\mu} X_{, b}^{v}\right) \\
= & 2 \gamma m r^{2} G^{3}\left(3-5 r^{4} \gamma^{2}\right) \dot{r}\left(\delta X^{r_{1}}+\delta X^{r_{2}}\right) \\
& \times\left(\delta X^{\phi_{2}}-\delta X^{\phi_{1}}\right) .
\end{aligned}
$$

Using

$$
\begin{aligned}
& \left(\delta X^{r_{1}}+\delta X^{r_{2}}\right)\left(\delta X^{\phi_{2}}-\delta X^{\phi_{1}}\right) \\
& \quad=\left[\left(n_{2}^{r_{1}}+n_{2}^{r_{2}}\right) \delta X_{2}+\left(n_{3}^{r_{1}}+n_{3}^{r_{2}}\right) \delta X_{3}\right]\left(n_{8}^{\phi_{1}}-n_{8}^{\phi_{2}}\right) \delta X_{8},
\end{aligned}
$$

we find the final form for the equations of motion for the perturbations

$$
\begin{gathered}
\left(\square-\frac{1}{2 r^{2}}+\gamma^{2} r^{2} \mathcal{G}(1-6 \mathcal{G})\right) \delta X_{3}=0, \\
\left(\square-\frac{5 \kappa^{2}}{4 m^{2}} \frac{(1-2 \mathcal{G})^{2}}{r^{4} \mathcal{G}}-\gamma^{2} \frac{\kappa^{2}}{m^{2}}(1-6 \mathcal{G})\right) \\
\times \delta X_{2}+4 \gamma \kappa \dot{r} \mathcal{G}(8 \mathcal{G}-5) \delta X_{8}=0, \\
\left(\square-\frac{1}{2 r^{2}}-\frac{\gamma^{2}}{m^{2}}\left[(8-\mathcal{G}) \dot{r}^{2}-2 \kappa^{2} \mathcal{G}\right]\right) \\
\times \delta X_{8}+4 \gamma \kappa \dot{r} \mathcal{G}(8 \mathcal{G}-5) \delta X_{2}=0, \\
\square \delta X_{r}=0, \quad r=4,5,6,7,9 .
\end{gathered}
$$

Equation (58) shows that for $r=4,5,6,7$ and 9 the perturbations are stable so that we have to consider only $r=2,3$ and 8 .

From now on we will analyse the stability for a small deformation $\gamma \ll 1$. Keeping only the leading terms in $\gamma$ the equations for the perturbations reduce to

$$
\begin{aligned}
& \left(\square-\frac{1}{2 r^{2}}-5 \gamma^{2} r^{2}\right) \delta X_{3}=0, \\
& \left(\square-\frac{5 \kappa^{2}}{4 m^{2}} \frac{1}{r^{4}}\right) \delta X_{2}+12 \gamma \kappa \dot{r} \delta X_{8}=0, \\
& \left(\square-\frac{1}{2 r^{2}}\right) \delta X_{8}+12 \gamma \kappa \dot{r} \delta X_{2}=0 .
\end{aligned}
$$

We can now expand $\delta X_{i}$ as

$\delta X_{i}=\sum_{n=-\infty}^{\infty} e^{i n \sigma} F_{i}(\tau)$

and use $\square=\frac{1}{2 m r^{2}}\left(-\partial_{\tau}^{2}+\partial_{\sigma}^{2}\right)$ to get

$$
\begin{aligned}
& \left(\frac{\mathrm{d}^{2}}{\mathrm{~d} \tau^{2}}+n^{2}+m^{2}+10 \gamma^{2} m^{2} r^{4}\right) F_{3}=0, \\
& \left(\frac{\mathrm{d}^{2}}{\mathrm{~d} \tau^{2}}+n^{2}+\frac{5 \kappa^{2}}{2} \frac{1}{r^{2}}\right) F_{2}-24 \gamma \kappa m^{2} r^{2} \dot{r} F_{8}=0,
\end{aligned}
$$

$\left(\frac{\mathrm{d}^{2}}{\mathrm{~d} \tau^{2}}+n^{2}+m^{2}\right) F_{8}-24 \gamma \kappa m^{2} r^{2} \dot{r} F_{2}=0$.

In these equations $r$ is the unperturbed solution to (39) which is a periodic function of $\tau$. Then, by the Sturm theorem, $F_{3}$ oscillates for large $\tau$ so that the perturbation $\delta X_{3}$ is stable. We can handle $F_{2}$ and $F_{8}$ by expanding in $\gamma$ as $F_{2}=U_{2}+\gamma V_{2}$ 


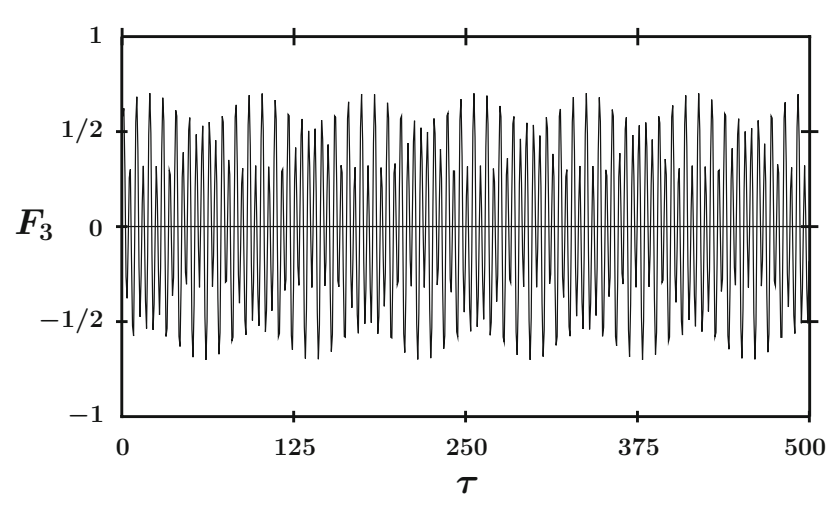

Fig. 2 Perturbation function $F_{3} \cdot \gamma=1 / 2, m=\kappa=1, n=0$

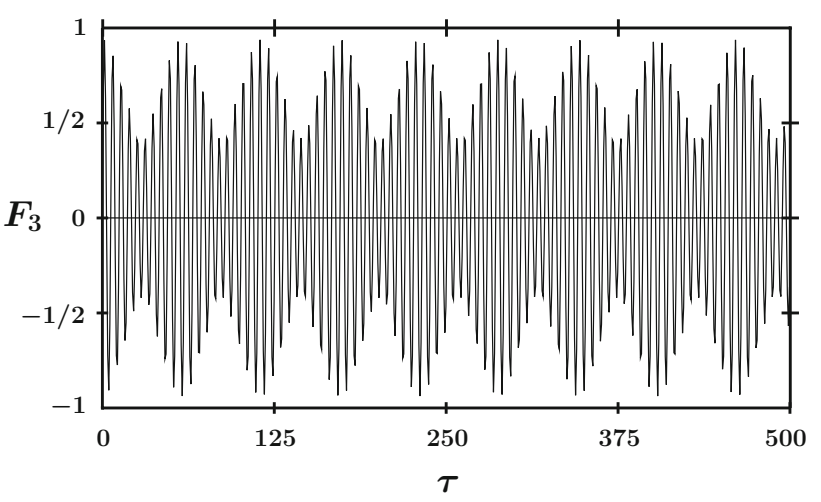

Fig. 3 Perturbation function $F_{3} \cdot \gamma=1 / 5, m=\kappa=1, n=0$

and $F_{8}=U_{8}+\gamma V_{8}$ to get

$$
\begin{aligned}
& \left(\frac{\mathrm{d}^{2}}{\mathrm{~d} \tau^{2}}+n^{2}+\frac{5 \kappa^{2}}{2} \frac{1}{r^{2}}\right) U_{2}=0, \\
& \left(\frac{\mathrm{d}^{2}}{\mathrm{~d} \tau^{2}}+n^{2}+m^{2}\right) U_{8}=0, \\
& \left(\frac{\mathrm{d}^{2}}{\mathrm{~d} \tau^{2}}+n^{2}+\frac{5 \kappa^{2}}{2} \frac{1}{r^{2}}\right) V_{2}-24 \kappa m^{2} r^{2} \dot{r} U_{8}=0, \\
& \left(\frac{\mathrm{d}^{2}}{\mathrm{~d} \tau^{2}}+n^{2}+m^{2}\right) V_{8}-24 \kappa m^{2} r^{2} \dot{r} U_{2}=0 .
\end{aligned}
$$

Then $U_{2}$ and $U_{8}$ are oscillatory for large $\tau$. In (68) the homogeneous solution for $V_{2}$ is also oscillatory for large $\tau$ as is the non-homogeneous term unless $U_{8}$ has some resonance frequency. This will happens whenever $m^{2}=\frac{5 \kappa^{2}}{2 r^{2}}$ or $r^{2}=\frac{5 \kappa^{2}}{2 m^{2}}$, but because $\frac{5 \kappa^{2}}{2 m^{2}}>r_{-}^{2}$ these values of $r$ cannot be reached so that there is no resonance. The same result holds for $V_{8}$ so the perturbations $\delta X_{2}$ and $\delta X_{8}$ are also stable. For arbitrary values of $\gamma$, (55) still shows that $\delta X_{3}$ is oscillatory but (56) and (57) could not be decoupled.

As a check that the perturbation is stable we will present some solutions of (63)-(69) in graphical form. To this end we need to consider a pulsating string solution of (39) in flat spacetime. As shown in [38] the condition to have an

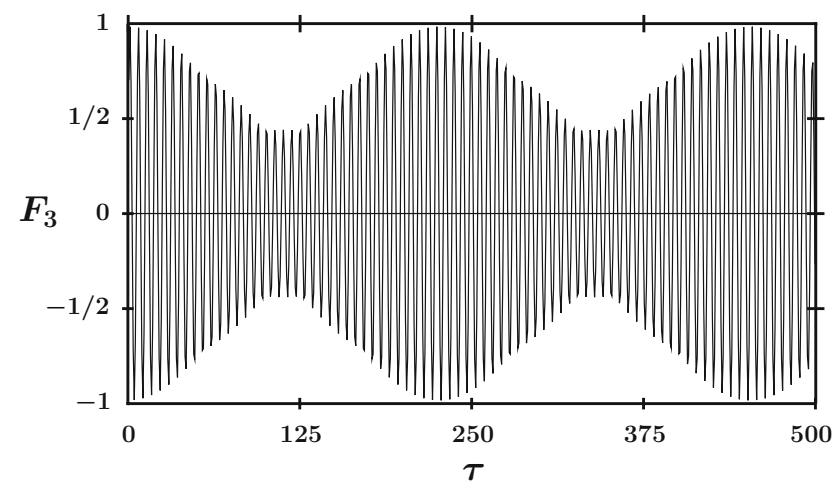

Fig. 4 Graphs of $F_{3}$ and $U_{2}(\tau)=\Re\left[\sec ^{2 p} m \tau{ }_{2} F_{1}\left(a, a^{*} ; c\right.\right.$; $\sec ^{2}$ $m \tau)] \cdot \gamma=1 / 10, m=\kappa=1, n=0$

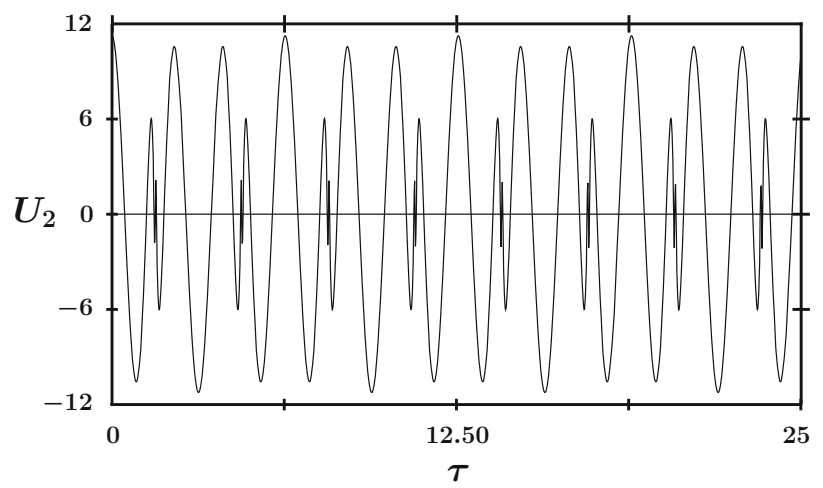

Fig. 5 Graphs of $F_{3}$ and $U_{2}(\tau)=\Re\left[\sec ^{2 p} m \tau \quad{ }_{2} F_{1}\left(a, a^{*} ; c ; \sec ^{2}\right.\right.$ $m \tau)] . p=3 / 2, m=1$

oscillatory motion is $2 \gamma \kappa^{2} \leq m^{2}$ and a solution of (39) in flat spacetime can be taken as $r(\tau)=\frac{\kappa}{m}|\cos m \tau|$. Using this solution in (63) we plot the mode $F_{3}$ in Figs. 2, 3 and 4. In these figures, we have for the deformation $\gamma=1 / 2,1 / 5$ and $1 / 10$, while all other parameters, the winding number $m$, the mode $n$ of the perturbation and the stringy energy $\kappa$, are fixed. In these cases the perturbation amplitude modulates and is stable.

We now turn our attention to (66). Since $r(\tau)$ is periodic the term in $1 / r^{2}$ diverges whenever $r$ vanishes and could cast doubts about the stability. In fact, when plotting $U_{2}$ we get warnings that there is some problem at $\tau=\pi /(2 \mathrm{~m})$. The same sort of problem appears in (64) and (65) or in (68) and (69). Our previous arguments, however, show that there is nothing special in those points. Since (66) is an ordinary differential equation we can solve it. Changing variables to $y=\sec ^{2}(m \tau)$ and taking $U_{2}(y)=y^{\frac{n}{2 m}} G(y)$ we find that $G(y)$ satisfies the hypergeometrical differential equation so that

$$
\begin{aligned}
U_{2}(\tau) & =\sec ^{n / 2 m}(m \tau)_{2} F_{1}\left(a, a^{*} ; c ; \sec ^{2}(m \tau)\right), \\
a & =\frac{n}{2 m}+\frac{1}{4} \pm \frac{3}{4} i, \quad c=\frac{n}{m}+1 .
\end{aligned}
$$




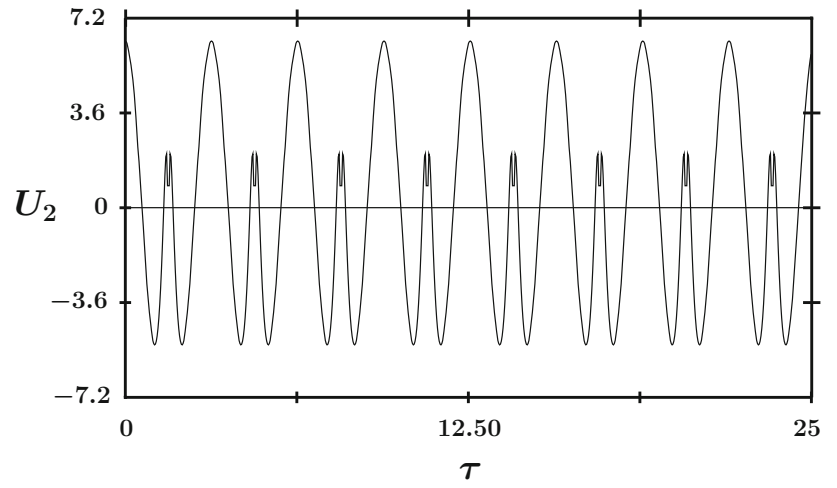

Fig. 6 Graphs of $U_{2}(\tau)=\Re\left[\sec ^{2 p} m \tau{ }_{2} F_{1}\left(a, a^{*} ; c\right.\right.$; $\left.\left.\sec ^{2} m \tau\right)\right]$. $p=m=1$

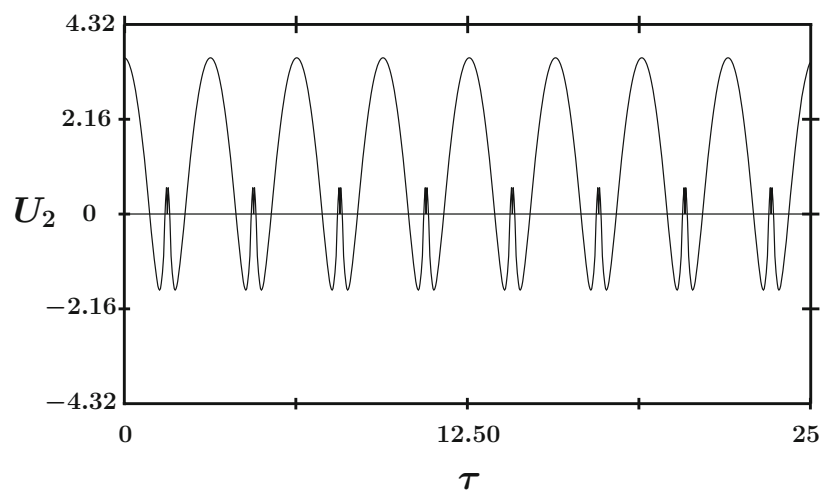

Fig. 7 Graphs of $U_{2}(\tau)=\Re\left[\sec ^{2 p} m \tau{ }_{2} F_{1}\left(a, a^{*} ; c\right.\right.$; $\left.\left.\sec ^{2} m \tau\right)\right]$. $p=0, m=1$

In Figs. 4, 5 and 6 we plot $U_{2}$ for some values of $m$ and $n$, showing that it is well behaved everywhere. Notice also the amplitude of $U_{2}$ modulates so that the $\gamma$ independent part of the perturbation of the mode $F_{2}$ is also stable. The $\gamma$ independent part of $F_{8}$, that is, $U_{8}$, is also stable since it satisfies (67). Then the homogeneous solutions for $V_{2}$ and $V_{8}$ in (68) and (69) are stable and the particular solutions, which involve $U_{8}$ and $U_{2}$, respectively, are also stable unless there is some resonance frequency (Fig. 7). But as discussed previously, this cannot happen so that the full solutions for $V_{2}$ and $V_{8}$ are also stable.

\section{Conclusions}

We have applied the WKB method to compute the tunnelling amplitude for an oscillating string in deformed Minkowski spacetime. As expected it is proportional to the string energy and vanishes when the deformation goes to zero. We have also shown that for small deformation the classical pulsating string is stable. It is well known that pulsating strings in $A d S_{5} \times S^{5}$ are dual to operators composed of nonholomorphic products of scalar fields [20,23,48,49], but the theory corresponding to the deformed Minkowski spacetime is not known. Since the string tunnelling represents an instability of the system it would be very interesting to see what happens on the other side of the correspondence.

Acknowledgments Sergio Giardino is supported by CNPq Grant 206383/2014-2 and thanks Prof. Paulo Vargas Moniz and the Center for Mathematics and Applications of the Beira Interior University for hospitality. The work of Victor Rivelles is supported by FAPESP Grant 2014/18634-9.

Open Access This article is distributed under the terms of the Creative Commons Attribution 4.0 International License (http://creativecomm ons.org/licenses/by/4.0/), which permits unrestricted use, distribution, and reproduction in any medium, provided you give appropriate credit to the original author(s) and the source, provide a link to the Creative Commons license, and indicate if changes were made.

Funded by SCOAP ${ }^{3}$.

\section{References}

1. N. Beisert, C. Ahn, L.F. Alday, Z. Bajnok, J.M. Drummond et al., Review of AdS/CFT integrability: an overview. Lett. Math. Phys. 99, 3-32 (2012). arXiv: 1012.3982

2. R.G. Leigh, M.J. Strassler, Exactly marginal operators and duality in four-dimensional $N=1$ supersymmetric gauge theory. Nucl. Phys. B 447, 95-136 (1995). arXiv:hep-th/9503121

3. A. Mauri, S. Penati, A. Santambrogio, D. Zanon, Exact results in planar $N=1$ superconformal Yang-Mills theory. JHEP 0511, 024 (2005). arXiv:hep-th/0507282

4. O. Lunin, J.M. Maldacena, Deforming field theories with $U(1) \times$ $U(1)$ global symmetry and their gravity duals. JHEP 0505, 033 (2005). arXiv:hep-th/0502086

5. S. He, J.-B. Wu, Note on Integrability of marginally deformed ABJ(M) theories. JHEP 1304, 012 (2013). arXiv:1302.2208

6. R. Roiban, On spin chains and field theories. JHEP 0409, 023 (2004). arXiv:hep-th/0312218

7. D. Berenstein, S.A. Cherkis, Deformations of $N=4 \mathrm{SYM}$ and integrable spin chain models. Nucl. Phys. B 702, 49-85 (2004). arXiv:hep-th/0405215

8. S. Frolov, R. Roiban, A.A. Tseytlin, Gauge-string duality for superconformal deformations of $N=4$ super Yang-Mills theory. JHEP 0507, 045 (2005). arXiv:hep-th/0503192

9. S. Frolov, Lax pair for strings in Lunin-Maldacena background. JHEP 0505, 069 (2005). arXiv:hep-th/0503201

10. N. Beisert, R. Roiban, Beauty and the twist: The Bethe ansatz for twisted $N=4$ SYM. JHEP 0508, 039 (2005). arXiv:hep-th/0505187

11. N. Gromov, V. Kazakov, P. Vieira, Exact spectrum of anomalous dimensions of planar $N=4$ supersymmetric Yang-Mills theory. Phys. Rev. Lett. 103, 131601 (2009). arXiv:0901.3753

12. C. Ahn, Z. Bajnok, D. Bombardelli, R.I. Nepomechie, Finite-size effect for four-loop Konishi of the $\beta$-deformed $N=4$ SYM, Phys. Lett. B 693, 380 (2010). arXiv:1006.2209

13. G. Arutyunov, M. de Leeuw, S.J. van Tongeren, Twisting the mirror TBA. JHEP 1102, 025 (2011). arXiv: 1009.4118

14. C. Ahn, M. Kim, B.-H. Lee, Worldsheet S-matrix of beta-deformed SYM. Phys. Lett. B 719, 458-463 (2013). arXiv:1211.4506

15. J. Fokken, C. Sieg, M. Wilhelm, The complete one-loop dilatation operator of planar real beta-deformed $N=4$ SYM theory, arXiv: 1312.2959

16. J.-B. Wu, Multi-spin strings in $A d S_{4} \times C P^{3}$ and its $\beta$-deformations. Nucl. Phys. B 873, 260-274 (2013). arXiv:1208.0389 
17. H. de Vega, A. Larsen, N.G. Sanchez, Semiclassical quantization of circular strings in de Sitter and anti-de Sitter space-times. Phys. Rev. D 51, 6917-6928 (1995). arXiv:hep-th/9410219

18. S. Gubser, I. Klebanov, A.M. Polyakov, A Semiclassical limit of the gauge/string correspondence. Nucl. Phys. B 636, 99-114 (2002). arXiv:hep-th/0204051

19. J.A. Minahan, Circular semiclassical string solutions on $\operatorname{AdS}(5) \times$ S(5). Nucl. Phys. B 648, 203-214 (2003). arXiv:hep-th/0209047

20. J. Engquist, J. Minahan, K. Zarembo, Yang-Mills duals for semiclassical strings on $\operatorname{AdS}(5) \times \mathrm{S}(5)$. JHEP 0311, 063 (2003). arXiv:hep-th/0310188

21. A. Khan, A. Larsen, Spinning pulsating string solitons in $A d S_{5} \times$ $S^{5}$. Phys. Rev. D 69, 026001 (2004). arXiv:hep-th/0310019

22. G. Arutyunov, J. Russo, A.A. Tseytlin, Spinning strings in $\operatorname{AdS}(5)$ $\times S^{* *}$ 5: new integrable system relations. Phys. Rev. D 69, 086009 (2004). arXiv:hep-th/0311004

23. M. Kruczenski, A.A. Tseytlin, Semiclassical relativistic strings in S**5 and long coherent operators in $N=4$ SYM theory. JHEP 0409, 038 (2004). arXiv:hep-th/0406189

24. M. Smedback, Pulsating strings on AdS(5) $\times$ S**5. JHEP 0407, 004 (2004). arXiv:hep-th/0405102

25. K.L. Panigrahi, P.M. Pradhan, On rotating and oscilla ting four-spin strings in $A d S_{5} \times S^{5}$. JHEP 1211, 053 (2012). arXiv: 1206.4920

26. P.M. Pradhan, K.L. Panigrahi, Pulsating strings with angular momenta. Phys. Rev. D 88, 086005 (2013). arXiv:1306.0457

27. D. Arnaudov, R.C. Rashkov, Three-point correlation functions from pulsating strings in $\mathrm{AdS}_{5} \times S^{5}$, arXiv: 1509.0283

28. B. Chen, J.-B. Wu, Semi-classical strings in $\operatorname{AdS}(4) \times \mathrm{CP}^{* * 3}$. JHEP 0809, 096 (2008). arXiv:0807.0802

29. H. Dimov, R. Rashkov, On the pulsating strings in $\mathrm{AdS}(4) \times \mathrm{CP}^{* * 3}$. Adv. High Energy Phys. 2009, 953987 (2009). arXiv:0908.2218

30. H. Dimov, R. Rashkov, Generalized pulsating strings. JHEP 0405 , 068 (2004). arXiv:hep-th/0404012

31. N. Bobev, H. Dimov, R. Rashkov, Pulsating strings in warped $\operatorname{AdS}(6) \times S^{* *} 4$ geometry, arXiv:hep-th/0410262

32. D. Arnaudov, H. Dimov, R. Rashkov, On the pulsating strings in Ad $S_{5} \times T^{1,1}$, arXiv:1006.1539

33. D. Arnaudov, H. Dimov, R. Rashkov, On the pulsating strings in Sasaki-Einstein spaces. AIP Conf. Proc. 1301, 51-58 (2010). arXiv: 1007.3364
34. A. Banerjee, K.L. Panigrahi, On the rotating and oscillating strings in $\left(\mathrm{AdS}_{3} \times \mathrm{S}^{3}\right)_{\kappa}$. JHEP 1409, 048 (2014). arXiv: 1406.3642

35. C. Cardona, Pulsating strings from two dimensional CFT on $\left(T^{4}\right)^{N} / S(N)$. Nucl. Phys. B 893, 512-524 (2015). arXiv: 1408.5035

36. K.L. Panigrahi, P.M. Pradhan, M. Samal, Pulsating strings on $\left(A d S_{3} \times S^{3}\right)$. JHEP 03, 010 (2015). arXiv:1412.6936

37. A. Banerjee, K. L. Panigrahi, M. Samal, A note on oscillating strings in $A d S_{3} \times S^{3}$ with mixed three-form fluxes, arXiv: 1508.03430

38. S. Giardino, V.O. Rivelles, Pulsating strings in Lunin-Maldacena backgrounds. JHEP 1107, 057 (2011). arXiv:1105.1353

39. M. Beccaria, G. Dunne, G. Macorini, A. Tirziu, A. Tseytlin, Exact computation of one-loop correction to energy of pulsating strings in $A d S_{5} \times S^{5}$. J. Phys. A 44, 015404 (2011). arXiv:1009.2318

40. B. Berg, Glueballs, string tension, tunneling and deconfinement. Nucl. Phys. Proc. Suppl. 4, 6-11 (1988)

41. R. Brustein, B.A. Ovrut, Stringy instantons. Phys. Lett. B 309, 45-52 (1993). arXiv:hep-th/9209045

42. J. Lee, P.F. Mende, Semiclassical tunneling in $(1+1)$ dimensional string theory. Phys. Lett. B 312, 433-440 (1993). arXiv:hep-th/9211049

43. L. Zhao, Tunnelling through black rings. Commun. Theor. Phys. 47, 835-842 (2007). arXiv:hep-th/0602065

44. A. Monin, M. Voloshin, Breaking of a metastable string at finite temperature. Phys. Rev. D 78, 125029 (2008). arXiv:0809.5286

45. S. Frolov, A.A. Tseytlin, Quantizing three spin string solution in AdS(5) $\times$ S**5. JHEP 0307, 016 (2003). arXiv:hep-th/0306130

46. A. Khan, A. Larsen, Improved stability for pulsating multispin string solitons. Int. J. Mod. Phys. A 21, 133-150 (2006). arXiv:hep-th/0502063

47. A. Larsen, V.P. Frolov, Propagation of perturbations along strings. Nucl. Phys. B 414, 129-146 (1994). arXiv:hep-th/9303001

48. N. Beisert, J. Minahan, M. Staudacher, K. Zarembo, Stringing spins and spinning strings. JHEP 0309, 010 (2003). arXiv:hep-th/0306139

49. J.A. Minahan, Higher loops beyond the SU(2) sector. JHEP 0410 , 053 (2004). arXiv:hep-th/0405243 